\title{
Enhancement of Photonic Crystal All-Optical Diode Efficiency with a Subwavelength Layer
}

\author{
K. JAMSHIDI-GHALEH ${ }^{a}$, Z. SAFARI $^{b}$ AND R. TANAVAR ${ }^{c}$ \\ ${ }^{a}$ Department of Physics and Research Group of Processing and Communication, Azarbaijan Shahid Madani \\ University, Tabriz, Iran \\ ${ }^{b}$ Department of Laser-Optics, University of Bonab, Azarbaijan-e Shargi, Bonab, Iran \\ ${ }^{c}$ Sama Technical and Vocational Training College, Islamic Azad University, Tabriz branch, Tabriz, Iran
}

\begin{abstract}
In this paper, the effect of a subwavelength thin layer on all-optical diode action in a one-dimensional photonic crystal is investigated. The photonic multilayer structure is considered as $(\mathrm{BA})^{6}(\mathrm{AB})^{6} \mathrm{C}(\mathrm{AABB})^{6}$, where layers $\mathrm{A}$ and $\mathrm{B}$ are nonlinear and linear materials respectively, and $\mathrm{C}$ is a thin layer with positive or negative-index material. The results are shown that by introducing a thin layer with different optical properties the all-optical diode efficiency is controllable. In our numerical results, the transmission contrast of the structure, without the thin layer is obtained as 0.63 , with a positive index material layer nearly zero and with a negative index material thin layer about 0.75 .
\end{abstract}

DOI: 10.12693/APhysPolA.123.212

PACS: 42.70.Qs

\section{Introduction}

Photonic crystals (PCs), firstly introduced by Yablonovich [1] and John [2], are periodic structures that are designed to control and manipulating of electromagnetic waves. The main characteristics of a PC structure is the photonic band gap where electromagnetic waves with frequency within the gap cannot propagate through the structure. In last two decades, due to special electromagnetic performance and potential applications of the $\mathrm{PCs}$, more attentions are attracted to design the devices capable to control the photons [3-6]. Optical bistability (OB) is one of the important ways to manipulating of photon's transportations and can be used to design all-optical switching $[7,8]$. OB can be induced by dynamic shifting of the band edge or resonant modes $[9,10]$. Inouye and Kanemitsu experimentally observed mode shifting and opened the new fields in application of PCs in the nonlinear optical devices [11].

The bistability of $1 \mathrm{D}$ PCs containing negative-index materials has also attracted much attention. The positive-negative-index periodic structures, containing a single nonlinear defect, have demonstrated tunable bistable switching and transmission [12]. It has been reported that introducing of a positive or negative-refractive index subwavelength thin layer in a $1 \mathrm{D} P C$ structure significantly affects the bistability threshold $[13,14]$. The OB performance of a spatially asymmetric $\mathrm{PC}$ structure together with anisotropy of the field intensity distribution in layers puts the structure to be a device that is called all-optical diode (AOD). Such a device permits to pass an electromagnetic wave from one side of the structure but not from other side. AOD is an electronic analogue of the electric diode that passes electricity from one side only. AOD is a spatially nonreciprocal device that plays important role in all optical signal processing. In the last years, most attentions are directed to design new AOD structures or to optimize its efficiency. AOD is characterized by the transmission contrast $\eta=\left(T_{\mathrm{L}}-T_{\mathrm{R}}\right) /\left(T_{\mathrm{L}}+T_{\mathrm{R}}\right)$ where $T_{\mathrm{L}}$ and $T_{\mathrm{R}}$ are left and right incident transmissions, respectively.

It has been shown that AOD performance can be achieved from a simple photonic multilayer of $(\mathrm{AB})^{m}(\mathrm{BA})^{n}(\mathrm{BBAA})^{k}$ and the effect of different period numbers $m, n$, and $k$ on AOD efficiency has been investigated [15]. In this paper, we consider this $\mathrm{PC}$ structure for $m=n=k=6$ that is: $(\mathrm{BA})^{6}(\mathrm{AB})^{6} \mathrm{C}(\mathrm{AABB})^{6}$ with an inserted subwavelength thin layer denoted by $\mathrm{C}$. Thickness of the thin layer is a fraction of wavelength $(\lambda / 8)$. The refractive index of thin layer can be conventional positive-index dielectric material (PIDM) or negative index meta-material (NIMM). We have investigated effect of the thin layer on AOD contrast. In our study all of the layers A and B are nonlinear and linear material, respectively. The well-known transfer matrix method [16] is used to analysis of the TE electromagnetic wave propagation through the structure. According to this method, the normally incident electric $E$ and magnetic $H$ fields at the opposite sides of each single layer can be related in the following way:

$$
\left(\begin{array}{c}
E \\
\mathrm{i} H
\end{array}\right)_{j}=M_{j}\left(\begin{array}{c}
E \\
\mathrm{i} H
\end{array}\right)_{j+1},
$$

here

$$
M_{j}=\left[\begin{array}{cc}
\cos \left(\frac{n_{j} d_{j} \omega}{c}\right) & n_{j}^{-1} \sin \left(\frac{n_{j} d_{j} \omega}{c}\right) \\
-n_{j} \sin \left(\frac{n_{j} d_{j} \omega}{c}\right) & \cos \left(\frac{n_{j} d_{j} \omega}{c}\right)
\end{array}\right],
$$

where $d_{j}$ is the thickness of the layer with refractive in$\operatorname{dex} n_{j}, \omega$ is frequency and $c$ the speed of light. We will assume that all materials are dispersionless and the refractive indices of the layers are frequency independent. 
The total transfer matrix of the whole structure can be obtained by multiplying together all transfer matrices of subsequent layers

$$
M=M_{1} M_{2} \ldots M_{N-1} M_{N}
$$

and the transmission spectrum in terms of the matrix elements is given by

$$
T(\omega)=\frac{4}{\left|M_{11}+M_{22}+\mathrm{i}\left(M_{21}-M_{21}\right)\right|^{2}} .
$$

\section{Results and discussions}

In the numerical calculations the intensity dependent refractive index of the layer $\mathrm{A}$ is considered as $n_{\mathrm{A}}^{\mathrm{NL}}=$ $n_{\mathrm{A}}\left(1+\chi_{\mathrm{A}}^{(3)}|E|^{2} / 2\right)$, where $n_{\mathrm{A}}$ is the linear refractive index and $\chi_{\mathrm{A}}^{(3)}$ the 3 th-order nonlinear susceptibility. Layer $\mathrm{B}$ is a dielectric medium with linear refractive index of $n_{\mathrm{B}}=2.3$ and $n_{\mathrm{C}}= \pm 1.55$ are used to refractive index value of the layer $C$. Designed wavelength is used as $\lambda=572 \mathrm{~nm}$ and the condition of $n_{\mathrm{B}} d_{\mathrm{B}}=n_{\mathrm{A}} d_{\mathrm{A}}=\lambda / 4$ is applied for optical thickness of the both layers $\mathrm{A}$ and $\mathrm{B}$. The thickness of layer $\mathrm{C}$ is taken to be $\lambda / 8$ (subwavelength). Figure 1a shows the normalized field distribution inside the layers for left incident at three cases; without the thin layer (blue), with a PIDM (red) and a NIMM thin layer (green). As it is seen, for the structure containing NIMM thin layer the field localization is stronger than for the PIDM. Figure 1b shows the transmission versus of the input intensity (hysteresis) from the structure for the same condition. It is seen that for the NIMM thin layer, OB threshold is reduced and for PNDM it is increased. The results of Fig. 1 indicate that the nonlinear modifications and hence dynamic variations in the case of NIMM thin layer would be greater than the PIDM.
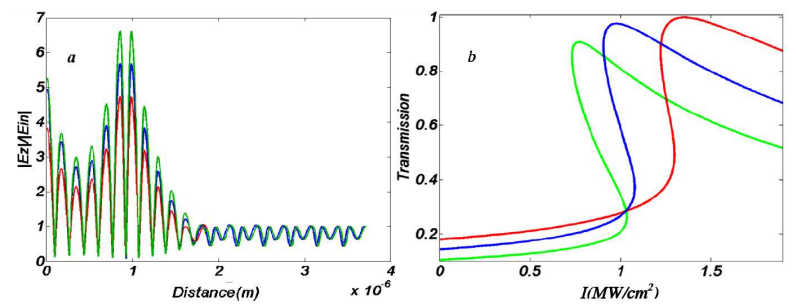

Fig. 1. Normalized field distribution inside the structure (a) and $\mathrm{OB}$ threshold variation (b) with introducing of a PIDM and NIMM in the PC structure. Blue: without, green: with NIMM and red: with PIDM thin layer.

Since the OB threshold is proportional to the field intensity inside the localized region it is predictable that the OB threshold in the case of NIMM would be lower than the PIDM one. Figure $2 \mathrm{a}-\mathrm{c}$ shows the hysteresis of transmission at three mentioned cases from the left to right $(\mathrm{L}-\mathrm{R})$ and right to left $(\mathrm{R}-\mathrm{L})$ beam incidences. It should be mentioned that difference between $\mathrm{L}-\mathrm{R}$ and $\mathrm{R}-\mathrm{L}$ of the $\mathrm{OB}$ curves displays the AOD property of a $\mathrm{PC}$ structure. It is clearly seen that with NIMM thin layer the AOD efficiency is better than the PIDM one. From the results of Fig. 2, the transmission contrast of the AOD without thin layer (a) is about 0.63 , with PIDM (b) about zero and with NIMM thin layer is (c) about 0.75 .

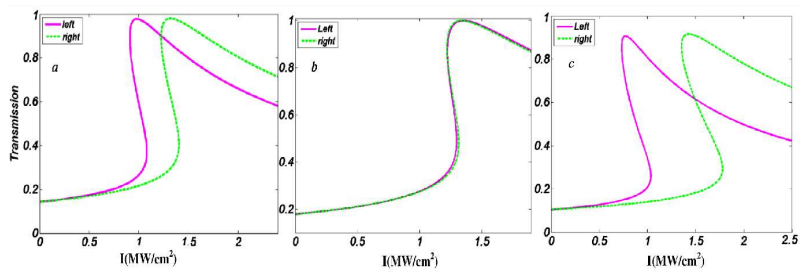

Fig. 2. Transmission through the structure versus incident intensity (OB) for left and right side incidents for three cases of (a) without, (b) with a PIDM, and (c) with a NIMM thin layer.

This is because that the positive and negative-index of the subwavelength layer have different effects on the linear defect mode, which strongly affects the OB threshold [15]. The linear defect mode shifts to lower frequencies in the case of positive-index layer and to higher frequencies in the case of negative-index layer one. The thin film of the subwavelength layer introduces an additional phase shift that affects the Bragg resonant condition. For negative index layer, the negative phase shift of negative refraction causes the linear defect mode of the whole structure to shift on higher frequencies, which, at the linear case, get strong the electric field intensity distribution in the nonlinear layers. So the threshold of OB decreases accordingly. In the case of a positive-index layer, opposite effects occur.

\section{Conclusions}

The effect of a subwavelength thin layer on AOD action in a one-dimensional multilayer photonic crystal structure of $(\mathrm{BA})^{6}(\mathrm{AB})^{6} \mathrm{C}(\mathrm{AABB})^{6}$ investigated, with all of layers $\mathrm{A}$ nonlinear medium, all of $\mathrm{B}$ layers linear and $\mathrm{C}$ a thin layer with positive or negative-index material. The results show that by introducing the thin layer with different optical properties in the photonic structure, the resonant peak, OB threshold and AOD efficiency can be controlled. We graphically illustrated that by a negative-index thin layer material AOD action is significantly modified. The transmission contrast in the absent thin layer is obtained about 0.63 , with PIDM nearly zero and with NIMM thin layer increased around 0.75 .

\section{References}

[1] E. Yablonovich, Phys. Rev. Lett. 58, 2059 (1987)

[2] S. John, Phys. Rev. Lett. 58, 2486 (1987)

[3] J.D. Joannopoulos, S.G. Johnson, J.N. Winn, R.D. Meade, Photonic Crystals: Modeling the Flow of Light, Princeton University Press, Princeton 2008.

[4] L. Jiusheng, Opt. Commun. 283, 2647 (2010). 
[5] R. Asadi, M. Malek-Mohammad, S. Khorsandi, Opt. Commun. 284, 2230 (2011)

[6] M.Y. Mahmoud, G. Bassou, A. Taalbi, Z.M. Chekrou, Opt. Commun. 285, 368 (2012)

[7] E. Wolf, Progress in Optics, North-Holland, Amsterdam 1984.

[8] J. He, M. Cada, IEEE J. Quantum Electron. QE-27, $1182(1991)$

[9] P. Tran, Opt. Lett. 21, 1138 (1996)

[10] R. Wang, J. Dong, D.Y. Xing, Phys. Rev. E 55, 6301 (1997)

[11] H. Inouye, Y. Kanemitsu, Appl. Phys. Lett. 82, 1155 (2003)
[12] M.W. Feisem, I.V. Shadrivov, Y.S. Kivshar, Appl. Phys. Lett. 85, 1451 (2004)

[13] P. Hou, Y. Chen, J. Shi, Q. Kong, L. Ge, Q. Wang, Appl. Phys. A 91, 41 (2008)

[14] P. Hou, Y. Chen, J. Shi, M. Shen, X. Chen, Q. Wang, Europhys. Lett. 81, 6401 (2008)

[15] R. Wang, J. Dong, D.Y. Hing, Phys. Rev. E 55, 6301 (1997).

[16] P. Yeh, Optical Waves in Layered Media, Wiley, New York 2005 